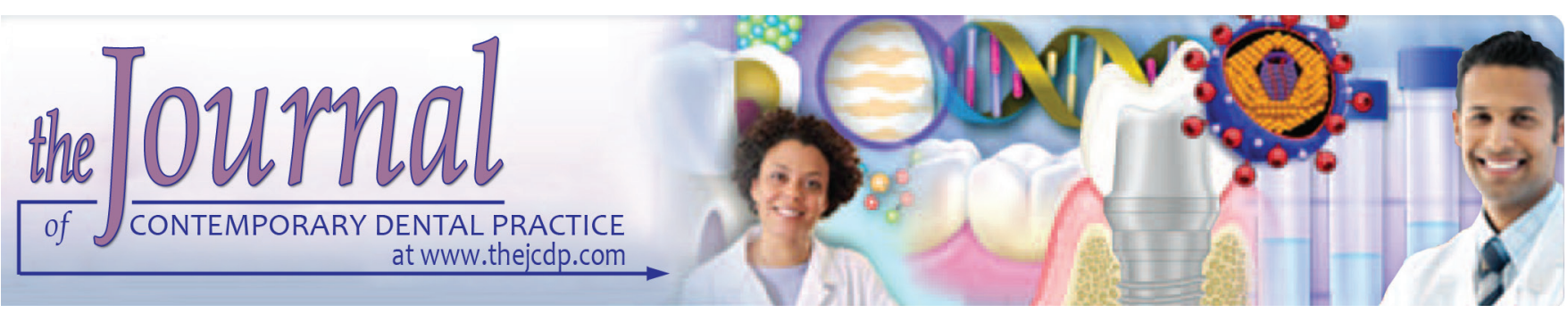

\title{
Pre-endodontic Post and Core Technique for Endodontic and Prosthodontic Treatment
}

\author{
${ }^{1}$ Keita Sasaki, ${ }^{2}$ Takatsugu Yamamoto, ${ }^{3}$ Tomoko Ikawa, ${ }^{4}$ Yuko Shigeta, ${ }^{5}$ Shuji Shigemoto, ${ }^{6}$ Eriko Ando \\ ${ }^{7}$ Takumi Ogawa, ${ }^{8}$ Keisuke Ihara
}

\begin{abstract}
Introduction: Displacement of provisional fixed prostheses may result in undesirable and embarrassing outcomes in dental treatments, especially in endodontic treatment. Development of certain counter measures has been necessary to avoid such discomforts.
\end{abstract}

Aim: The aim of this report was to propose a pre-endodontic post and core technique to achieve smooth progress of the treatment.

Materials and methods: The patient was a 59-year-old male diagnosed with an infraocclusion caused by wear of his teeth. He received full mouth provisional fixed restorations for a complete oral rehabilitation. Displacement and fracture of the restorations frequently occurred during the observation period for the function of the restorations. Therefore, the pre-endodontic post and core technique was applied to the abutment teeth before their endodontic treatments were started. The technique consisted of three steps as follows: Step 1: Caries removal and dowel preparation were performed for the abutment teeth having apical periodontitis. Composite cores were indirectly fabricated, which had access holes for endodontic treatment. Step 2: The cores were bonded to the teeth. In endodontic treatment, rubber dam appliances were easily placed owing to the core, and proper tooth isolation was accomplished. Step 3: Fiberposts were bonded to the dowel holes through the access holes after the root canal filling. During endodontic treatment, displacement and/or fracture of the provisional restorations did not occur.

Conclusion: The pre-endodontic post and core technique was effective in obtaining improved retention of provisional

\footnotetext{
1,3-7Department of Fixed Prosthodontics, School of Dental Medicine, Tsurumi University, Yokohama, Japan

${ }^{2}$ Department of Operative Dentistry, School of Dental Medicine Tsurumi University, Yokohama, Japan

${ }^{8}$ Dental Technician Training Institute, School of Dental Medicine Tsurumi University, Yokohama, Japan

Corresponding Author: Takatsugu Yamamoto, Department of Operative Dentistry, School of Dental Medicine, Tsurumi University, Yokohama, Japan, Phone: +81455808550, e-mail: yamamoto-tk@tsurumi-u.ac.jp
}

restoration, appropriate isolation for endodontic treatment, and sufficient retention of the post and core.

Clinical significance: The pre-endodontic post and core technique is useful for avoiding the discomforts in dental treatments, namely, a smooth transition from endodontic to prosthodontic treatment can be achieved.

Keywords: Composites, Dental prosthesis, Post and core, Root canal treatment, Rubber dam.

How to cite this article: Sasaki K, Yamamoto T, Ikawa T, Shigeta Y, Shigemoto S, Ando E, Ogawa T, Ihara K. Pre-endodontic Post and Core Technique for Endodontic and Prosthodontic Treatment. J Contemp Dent Pract 2018;19(1):117-122.

\section{Source of support: Nil}

Conflict of interest: None

\section{INTRODUCTION}

Provisional restorations are important treatment procedures in fixed prosthodontic rehabilitation, particularly when the restorations are expected to function for extended periods of time and/or when additional therapy is required before completion of the rehabilitation. ${ }^{1}$ Provisional fixed restorations are generally made of polymeric materials, such as methyl methacrylate. The provisional restorations sometimes demonstrate fractures with heavy occlusal loading. The long-span restorations have been reported to fail even under normal masticatory functions. ${ }^{2}$ Failure of the restorations leads not only to patient discomfort, but also complicated and prolonged treatment. In endodontic treatment, lack of temporary sealing ranks the second most common factor among the factors contributing to continuous discomfort during the treatment. ${ }^{3}$ Some papers have reported that the temporary restorations facilitate rubber dam placement and temporary sealing of tooth during endodontic treatment. ${ }^{4,5}$ Several techniques have been proposed for the temporary restorations, such as temporary crown 
with hollow or removable posts, wall buildup using resin composite or amalgam, and use of copper or orthodontic bands. ${ }^{6-8}$ It is occasionally required to use provisional restorations of the post-crown type during endodontic therapy when little coronal tooth remains. However, the posts that are supposed to aid in retention of the restoration to the dowel hole are not always feasible because the post must be shorter than the dowel hole to secure a space for endomedication; difference in directions of dowel holes is apt to produce undercuts interfering in the placement of restoration in oral rehabilitation cases having multiple abutments. Furthermore, the repeated removal of the posts during endodontic treatment may weaken the remaining tooth structures, and the wedge effect of the post may cause root fractures.

Resin composite cores are built up in two ways: Directly or indirectly. Comparing the direct and indirect methods, the former reportedly demonstrated greater dentin bond strength, ${ }^{9}$ whereas the latter has an advantage of contouring the cores in proper taper because of the laboratory work. In this case report, we propose a technique for resin composite post and core in which the core is fabricated indirectly for proper contour and the fiberpost is bonded directly to dowel hole for bond strength.

\section{MATERIALS AND METHODS}

The patient was a 59-year-old male. He complained of masticatory disturbance due to an infraocclusion caused by wear of his teeth (Fig. 1A). Oral rehabilitation was planned for his treatment. The full mouth provisional restorations were placed to improve the occlusal relations before endodontic treatments. During the observation period for oral functions with the provisional restorations, the restorations demonstrated the displacement and/or fracture repeatedly (Fig. 1B). The patient had several teeth requiring endodontic treatments (Fig. 1C). Therefore, the pre-endodontic post and core technique was applied to the teeth. The technique consists of three steps as follows:

\section{Step 1: Fabrication of the Pre-endodontic Core}

Before endodontic treatment, dowel preparations were roughly performed in the abutment teeth after caries removal. The impression was taken using hydrocolloid impression materials, and the plaster casts were made. Figure 2A shows the fabrication steps of indirect composite cores on the casts. Small woodpicks were inserted into the dowel holes on which paraffin wax was thinly applied as a separator (Fig. 2Aa). A resin composite for core foundation (BeautiCore, Shofu, Kyoto, Japan) was built upon the cast (Fig. 2Ab). The composite was lightcured and the woodpicks were pulled out of the cast and composite so that the composite cores had access holes for the subsequent endodontic treatment (Fig. 2Ac). Adjustments of the contours of cores were performed on the cast according to a technique for an indirect composite core buildup method. ${ }^{10}$ The access holes were refilled with a silicone-based impression material and radial pins (herein after impression pin) to avoid inflow of luting composite into the dowel holes while cementing the cores to the abutment teeth (Fig. 2Ad-f). Subsequently, the provisional restorations fitting to the cores were fabricated before the next clinical step.

\section{Step 2: Adhesion of the Core}

Surfaces of the fabricated cores were air-blasted with $70 \mu \mathrm{m}$ alumina particles followed by cleaning with $30 \%$ phosphoric acid and treated with a silane coupling agent (Shofu Porcelain Primer, Shofu, Kyoto, Japan) for bonding to the abutment teeth.

The dowel holes were cleaned using a root canal cleaning brush and $25 \mu \mathrm{m}$ alumina slurry. A two-bottle one-step self-etch adhesive (BeautiDual Bond, Shofu, Kyoto, Japan) was applied to the holes according to the manufacturer's instructions. The silane-treated cores with the impression pins were bonded to the abutment teeth using the same resin composite described previously (Fig. 2B). After irradiating the luting composite in multiple directions, the impression pins were removed, and the composite was additionally irradiated through the access holes to secure the polymerization and adhesion. The provisional restorations were set to the abutment teeth with slight adjustment. Then, endodontic treatments could be carried out with proper rubber dam isolation through the access holes (Fig. 3). During the period of the endodontic treatments, there was neither displacement nor fracture of the provisional restorations.

\section{Step 3: Adhesion of the Fiberposts}

After endodontic treatment, the roughly prepared dowel holes were re-prepared and brushed to clean the walls of the holes. The silane (Shofu Porcelain Primer) was applied to the access holes of the cores followed by the application of the one-step self-etch adhesive (BeautiDual Bond) to the dowel holes. Fiberposts (BeautiCore Fiber Post, Shofu, Kyoto, Japan) were inserted and bonded to the dowel holes and the cores using the resin composite (BeautiCore, Fig. 4). Through this process, the post can be bonded to the root by the direct method providing the higher bond strength than the indirect method as mentioned in the introduction.

\section{DISCUSSION}

Remarkable progress in dental adhesive technology has pushed the limits of the application range of the adhesion. 

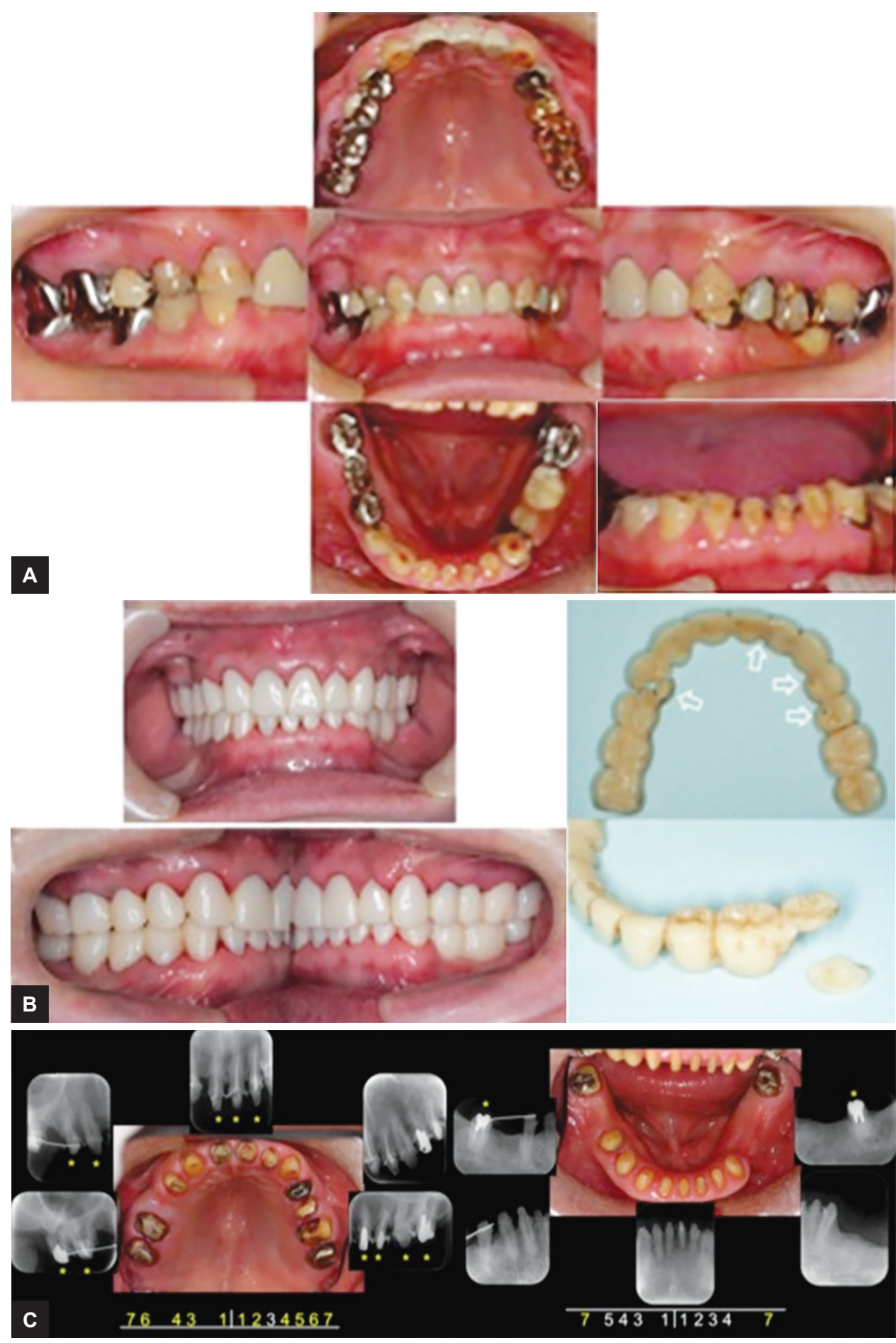

Figs $1 \mathrm{~A}$ to $\mathrm{C}$ : Intraoral and $\mathrm{X}$-ray findings: (A) Intraoral findings at initial visit; (B) provisional restorations: Left: Intraoral findings with provisional restorations, Right: Displacement and fracture of the provisional restorations; and $(\mathrm{C})$ intraoral and $\mathrm{X}$-ray findings after tooth preparations for the provisional restorations; yellow asterisks $\left(^{*}\right)$ in the $\mathrm{X}$-ray pictures indicate the requiring endodontic treatment

Resin composite is currently capable of bonding not only to tooth substrate, but also to several restorative materials, such as cured composite and ceramic. The pre-endodontic post and core technique is accomplished by taking the advantages of the adhesion. The adherends are radicular dentin, fiberpost, and cured composite.

\section{Placement of the Core}

Major roles of core buildups are retention of prosthesis and reinforcement of remaining tooth structure. The core presented here has an additional role of being an isolation wall for endodontic treatments. Endodontic treatments require proper isolation of the operating field 


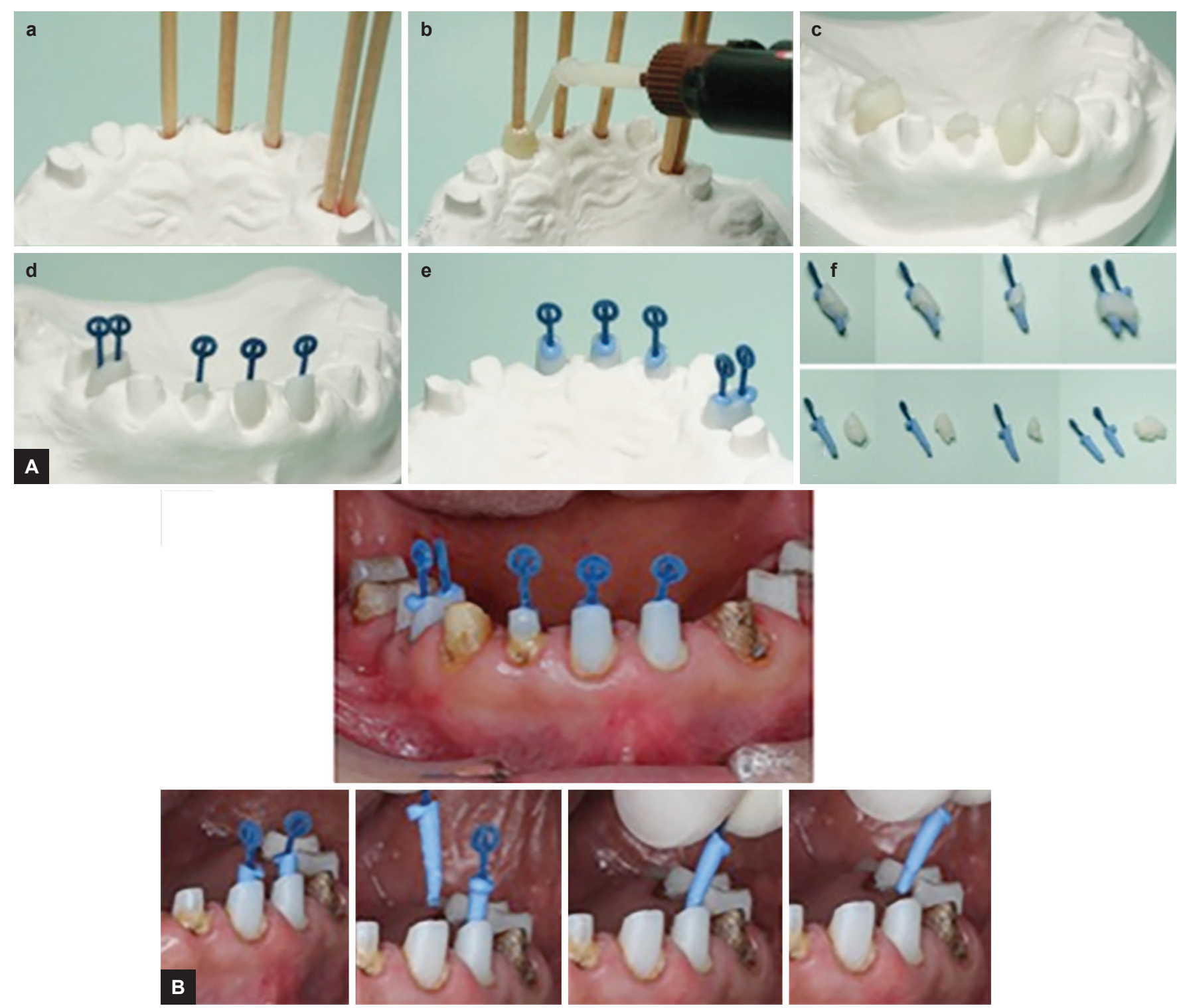

Figs 2A and B: Pre-endodontic cores: (A) Fabrication steps of the cores; and (B) the cores in the abutment teeth after bonding, the impression pins were pulled out
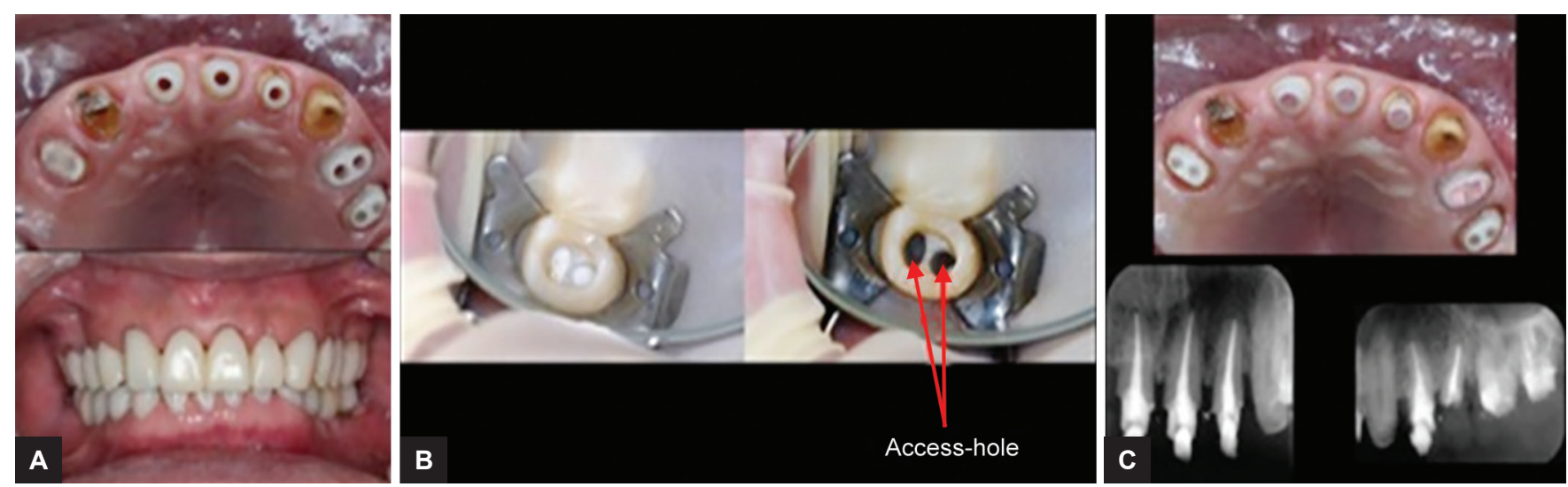

Figs $3 \mathrm{~A}$ to $\mathrm{C}$ : The endodontic treatments were carried out through the access-holes with proper rubber dam isolation

and sufficient sealing of root canals, and the rubber dam is the most common and effective appliance to obtain this isolation. The rubber dam has been reported to positively affect the treatment. ${ }^{3}$ Therefore, when endodontic treatment is planned for a structurally compromised tooth having subgingival margins, it is desirable to prepare the wall over which the rubber dam clamp is placed before treating the root canals. ${ }^{7}$ Placement of the pre-endodontic 

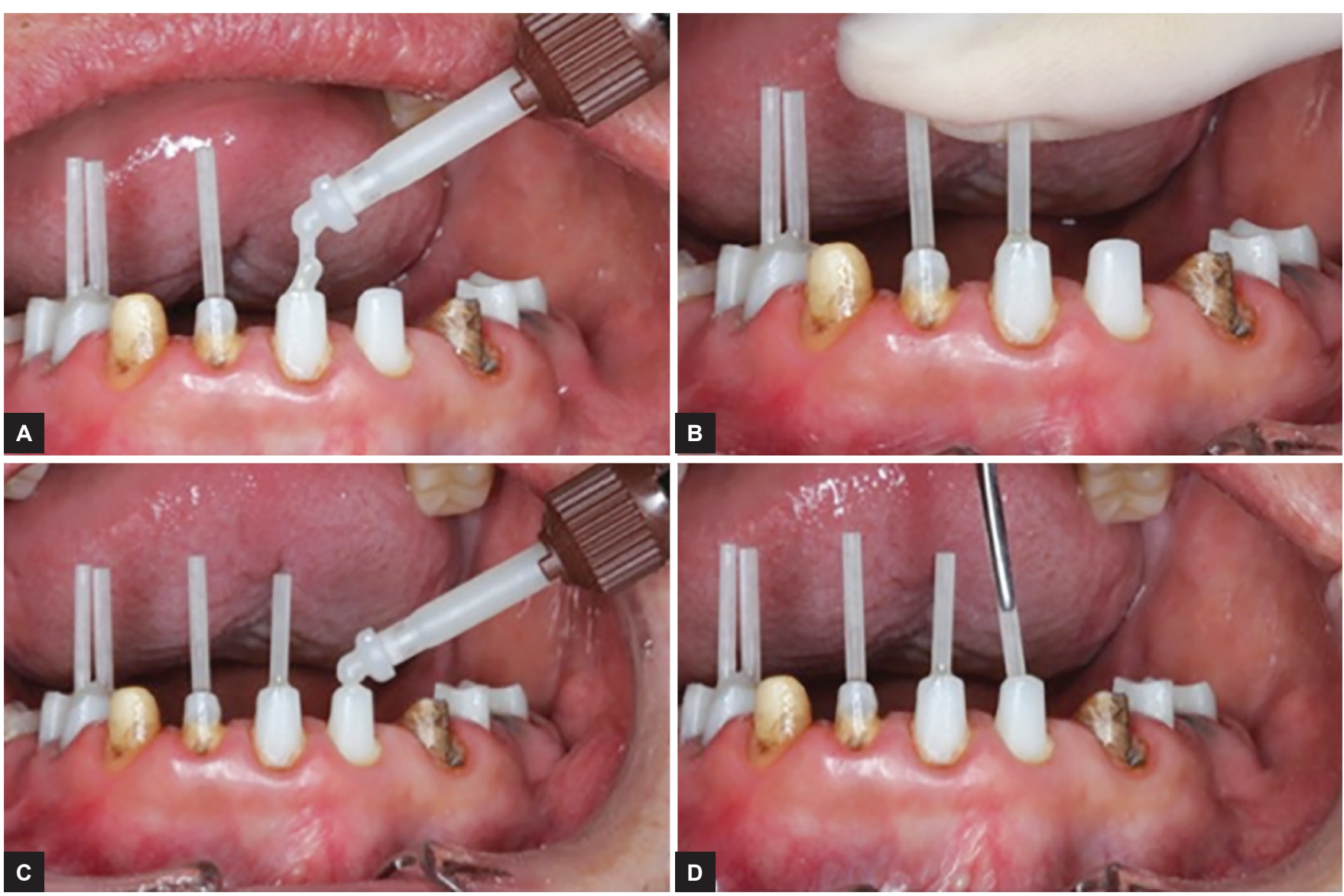

Figs 4A to D: Fiberposts were bonded to the dowel holes and the cores

core obviously facilitates the placement of the rubber dam. Resin composite for core foundation is a suitable material for the wall because of the improved adhesion to tooth and mechanical properties. ${ }^{8}$

Isolation walls are generally removed after endodontic treatment. However, our technique does not require their removal because the walls are resin composite cores used for the final restorations. This must contribute to the smoother progress of treatment, and in particular, to the oral rehabilitation in which multiple abutment teeth are manipulated.

To obtain sufficient sealing during endodontic treatment, temporary filling materials should be in a certain thickness. The minimum thickness has been reported to be $3.5 \mathrm{~mm}$ for a water-hardening temporary filling material. ${ }^{11}$ In this technique, sealing materials are filled in cylindrical holes (Fig. 3) penetrating the cores. The sealing ability is definitely improved due to the shape of the holes. In addition, provisional restorations are placed over the cores with temporary cement, indicating improved sealing can be achieved. Thus, the pre-endodontic core is able to fulfill multiple roles: Improved retention of provisional restorations, proper placement of rubber dam for tooth isolation, and sufficient sealing of root canals.

In placing the cores, securing a good adhesion between the core and tooth is essential for treatment success, as leakage at the bonded interface is almost clinically undetectable. Attention should be particularly paid to the following matters regarding the adhesion.

- Bonding procedures must be done steadily and accurately under appropriate moisture control.

- A certain magnitude of force is applied to the core when the rubber dam is placed. To avoid debonding of the core from the tooth, endodontic treatment should start a few days after the placement of the core because the bond strength of the composite improves with the postirradiation polymerization. ${ }^{12,13}$

\section{Adhesion of the Post}

Fiberposts are generally bonded to radicular dentin. However, in this technique, the posts are bonded to two substrates: Radicular dentin and the core (i.e., cured composite). In addition to tooth adhesives, silane coupling agents are needed for the adhesion, accordingly. ${ }^{14}$ There are two types of the silane coupling agents differing in their application ways; one is applicable by itself, and the other is mixed with an acidic solution, such as tooth adhesives, before application. With the former type, the silane coupling agent is applied to the internal surfaces of the access holes in the core first, and then, tooth adhesive is applied. With the latter agent, a mixture of the agent and an adhesive is applied to both the core and dentin. 
Both types of the agent significantly improve the adhesion and are clinically acceptable. ${ }^{15}$ Owing to the cores, appropriate moisture control can be easily performed during the bonding procedures of the post.

The operating field, namely, the areas for adhesion, is small because the only area is the internal surfaces of the post holes. More attention must be paid to the bonding procedures, such as the cleaning of the bonding surfaces and air-flowing the adhesives.

\section{CONCLUSION}

The pre-endodontic post and core technique is effective to obtain improved retention of provisional restoration and the core and facilitate rubber dam isolation. The technique achieves a smooth shift from endodontic treatment to prosthodontic treatment.

\section{Clinical Significance}

The pre-endodontic post and core technique is useful in avoiding discomfort in dental treatments and providing a smooth shift from endodontic to prosthodontic treatment.

\section{ACKNOWLEDGMENT}

Authors would like to thank Dr Asiri Jayawardena for providing suggestions on how to improve the use of English in the manuscript.

\section{REFERENCES}

1. Binkley CJ, Irvin PT. Reinforced heat-processed acrylic resin provisional restorations. J Prosthet Dent 1987 Jun;57(6):689-693.

2. Chen WC, Hung CC, Huang YC, Wang CK, Wang JC. Fracture load of provisional fixed partial dentures with long-span fiber-reinforced acrylic resin and thermocycling. J Dent Sci 2009 Mar;4(1):25-31.
3. Abbott PV. Factors associated with continuing pain in endodontics. Aust Dent J 1994 Jun;39(3):157-161.

4. Jensen AL, Abbott PV, Castro Salgado J. Interim and temporary restoration of teeth during endodontic treatment. Aust Dent J 2007 Mar;52(1 Suppl):S83-S99.

5. Samani SI, Harris WT. Provisional restorations for traumatically injured teeth requiring endodontic treatment. J Prosthet Dent 1980 Jul;44(1):36-39.

6. Chakalov I, Ivanova P, Apostolov N. Application of hollow posts for prosthetic provisionalisation and pre-endodontic build-up of severely destructed teeth. MedInform 2015 Jul;2:147-152.

7. Glickman, GN.; Pettiette, MT. Preparation for treatment. In: Cohen S, Hargreaves KM, editors. Pathways of the pulp. St. Louis (MO): Mosby Elsevier; 2006. p. 97-135.

8. Heydrich RW. Pre-endodontic treatment restorations. A modification of the "donut" technique. J Am Dent Assoc 2005 May;136(5):641-642.

9. Aksornmuang J, Nakajima M, Senawongse P, Tagami J. Effects of $\mathrm{C}$-factor and resin volume on the bonding to root canal with and without fibre post insertion. J Dent 2011 Jun;39(6):422-429.

10. Nagase DY, Takemoto S, Hattori M, Yoshinari M, Kawada E, Oda Y. Influence of fabrication techniques on retention force of fiber-reinforced composite posts. Dent Mater J 2005 Jun;24(2):280-285.

11. Webber RT, del Rio CE, Brady JM, Segall RO. Sealing quality of a temporary filling material. Oral Surg Oral Med Oral Pathol 1978 Jul;46(1):123-130.

12. Tarumi H, Imazato S, Ehara A, Kato S, Ebi N, Ebisu S. Postirradiation polymerization of composites containing bis-GMA and TEGDMA. Dent Mater 1999 Jul;15(4):238-242.

13. Talic YF. Immediate and 24-hour bond strengths of two dental adhesive systems to three tooth substrates. J Contemp Dent Pract 2003 Nov;4(4):28-39.

14. Moraes AP, Sarkis-Onofre R, Moraes RR, Cenci MS, Soares CJ, Pereira-Cenci T. Can silanization increase the retention of glass-fiber posts? A systematic review and meta-analysis of in vitro studies. Oper Dent 2015 Nov-Dec;40(6):567-580.

15. Staxrud F, Dahl JE. Silanising agents promote resin-composite repair. Int Dent J 2015 Dec;65(6):311-315. 